\title{
Conséquences d'une réduction du taux de matières azotées sur le niveau de consommation et les performances de croissance chez le porc selon l'équilibre en acides aminés et la concentration en énergie du régime
}

\author{
J. NOBLET et Y. HENRY \\ avec la collaboration technique de \\ G. Conseil, B. Magdelaine et Chantal Blondel \\ Station de Recherches sur l'Eletage des Porcs, \\ Centre National de Recherches Zootechniques, I.N.R.A., \\ $7835^{\circ}$ Jouty-en-Josas (France)
}

\section{Résumé}

Une expérience a été réalisée sur 72 porcs de race large White entre 20 et 1 oo $\mathrm{kg}$ de poids vif, afin d'étudier l'influence d'une réduction du taux de matières azotées dans la ration sur Ie niveau de consommation, les performances de croissance et les caractéristiques de composition corporelle, selon l'équilibre en acides aminés et la concentration en énergie du régime. Six lots de 12 animaux (6 mâles castrés, 6 femelles), nourris à volonté, sont mis en comparaison suivant un dispositif factoriel $2 \times 3: 2$ concentrations en énergie $(3$ too et 3 050 Kical d'énergie digestible $/ \mathrm{kg}$ d'aliment, respectivement à base de maîs et d'orge) et 3 niveaux azotés (l'un "normal ", correspondant aux recommandations habituelles, les deux autres réduits de $25 \mathrm{p}$. Ioo, avec supplémentation ou non en lysine et tryptophane).

Le remplacement du maïs par I'orge dans la ration aboutit à une consommation d'énergic digestible sensiblement équivalente, mais la vitesse de croissance est réduite et l'indice de consommation augmenté, principalement chez les mâles castrés (respectivement -.. I 2,5 et + I I p. Ioo) tandis que l'adiposité des carcasses est accrue.

La réduction globale du taux de matières azotées provoque une hyperphagie compensatrice chez les animaux recevant les régimes à base de maïs, mais non avec l'orge. Quelle que soit la céréale utilisée, le gain moyen pondéral et l'efficacité alimentaire sont abaissés, principalement chez les mâles castrés, tandis que l'état d'engraissement des animaux est plus élevé.

La surconsommation d'aliment constatée avec les régimes à base de maïs à taux réduit de matières azotées est maintenue après addition des acides aminés limitants, mais d'une façon moins marquée chez les femelles que chez les mâles castrés. Cependant, comparativement aux animaux recevant une ration à taux azoté normal, les performances de croissance sont sensiblement plus faibles et l'état d'engraissement est significativement plus élevé, notamment avec les régimes mails-tourteau de soja. Les résultats font enfin apparaître une réponse différente selon le sexe : les femelles sont plus sensibles aux apports azotés quantitatifs et qualitatifs que les mâles castrés; de plus, ces derniers réagissent d'une façon particulièrement défavorable à une diminution de la concentration en énergie du régime. 


\section{Introduction}

L'influence du taux de matières azotées sur les performances de croissance et la composition corporelle du porc, en relation avec la concentration en énergie du régime, a fait l'objet d'un grand nombre de travaux, tant en alimentation à volonté que dans les conditions du rationnement (HENRY et RERAT, I972). Lorsque le porc est nourri à volonté, il apparaît important de dissocier les effets propres du taux de protéines (azote indifférencié) et de l'équilibre en acides aminés sur le niveau d'ingestion d'aliment, ce qui n'a pas été le cas dans la plupart des travaux réalisés.

Grâce à une meilleure connaissance du besoin azoté qualitatif du porc en croissance et à la possibilité d'améliorer l'équilibre en acides aminés de la ration par la voie de la supplémentation, on peut envisager une réduction des normes azotées quantitatives, dans les conditions d'un équilibre optimal en acides aminés (Henry, Pion et Rerat, 1976).

Compte tenu de ces considérations, il nous a paru intéressant d'étudier la réponse du porc à une diminution de l'apport azoté global, après supplémentation en acides aminés limitants (lysine, tryptophane), selon la concentration en énergie du régime et dans le cas de l'alimentation à volonté.

\section{Matériel et méthodes}

Soixante-douze porcelets de race Large White et d'un poids vif moyen initial de $22,5 \mathrm{~kg}$ sont répartis en 2 groupes de 3 lots composés chacun de 12 animaux ( 6 mâles castrés et 6 femelles), suivant la méthode des blocs complets équilibrés. Le premier groupe (lots I, 2 et 3 ) reçoit des régimes à base de maiss riches en énergie (3 $400 \mathrm{Kcal}$ Energie Digestible : ED/kg d'aliment), le deuxième (lots 4, 5 et 6) étant soumis à des régimes à base d'orge $(3050 \mathrm{Kcal} \mathrm{ED} / \mathrm{kg})$. A l'intérieur de chaque groupe, on procède à une réduction du taux de matières azotées dans la ration, sans ou avec compensation par un apport supplémentaire de lysine et de tryptophane, facteurs limitants des régimes à base de maïs ou d'orge (SHARDA, Mahan et Wilson, I976; Henry, I977).

Les régimes des lots I et 4 sont normalement pourvus en matières azotées et en acides aminés, correspondant à 43,5 et 39,o g de matières azotées digestibles par Mcal ED, respectivement entre 20 et $50 \mathrm{~kg}$ de poids vif et au-delà de $50 \mathrm{~kg}$; l'utilisation digestive des protéines a été estimée à 87 et 83 p. Ioo, respectivement pour les régimes à base de maïs et d'orge (HeNRY, I976). Dans les lots 2 et 5, on procède à une réduction d'environ $25 \mathrm{p}$. Ioo de la teneur en matières azotées et, par suite en acides aminés, soit respectivement 39 et $33 \mathrm{~g}$ de matières azotées digestibles par Mcal ED au cours des deux périodes considérées. Dans les lots 3 et 6 , la réduction du taux de matières azotées, dans les mêmes proportions que dans les lots 2 et 5, est accompagnée d'une supplémentation en L-Lysine et L-Tryptophane. La composition des régimes est détaillée dans le tableau I.

Les animaux sont élevés en loges indıviduelles. L'aliment présenté sous forme de granulés est distribué à volonté, les consommations étant enregistrées quotidiennement.

Les porcs sont pesés toutes les deux semaines. A $100 \mathrm{~kg}$, ils sont abattus et, après un ressuyage de 24 heures, les carcasses sont soumises à la découpe parisienne, 
en complément des mesures habituelles (épaisseur du lard au dos et au rein, longueur de la carcasse).

Les résultats de consommation et de croissance pour la période $20-50 \mathrm{~kg}$ et la période totale 20-Ioo $\mathrm{kg}$, ainsi que les caractéristiques de composition corporelle, sont traités par l'analyse de variance, avec séparation des effets principaux (sexe, concentration en énergie du régime ou effet céréale, réduction du taux azoté sans ou avec supplémentation en acides aminés) et des effets d'interaction. Pour chaque céréale, des comparaisons multiples de moyennes sont effectuées.

\section{Résultats}

Sur les 72 animaux de départ, 4 n'ont pas terminé l'expérience (rachitisme et ulcère gastrique : 2 dans le lot 2 et 2 dans le lot 6). Sur l'ensemble des données, l'analyse de variance n'a pas permis de mettre en évidence d'interaction significative entre le sexe et la concentration énergétique du régime, confondue avec l'effet céréale. L'influence du sexe se traduit par une consommation d'énergie digestible et une vitesse de croissance plus faibles chez les femelles que chez les mâles castrés (7,3I Mcal ED/jour contre 7,98 et $700 \mathrm{~g} /$ jour contre 750 entre 20 et I $00 \mathrm{~kg}$ ). L'indice de consommation n'est toutefois pas modifié, que ce soit pendant la période de croissance ou sur l'ensemble de l'expérience. Il en résulte une adiposité moindre des femelles, le rapport longe/bardière s'élevant à I,92 au lieu de I,64 chez les mâles castrés. Les résultats de consommation et de croissance dans les intervalles de poids $20-50 \mathrm{~kg}$ et $20-100 \mathrm{~kg}$ sont rassemblés respectivement dans les tableaux 2 et 3 , tandis que l'évolution du niveau de consommation d'énergie digestible en fonction du poids vif moyen par lot et par sexe est décrite dans les figures i et 2 . Les caractéristiques finales des carcasses sont consignées dans le tableau 4 .

\section{I. - Influence de la concentration en énergie du régime (ou nature de la céréale)}

Pour l'ensemble des traitements, l'utilisation de régimes à base d'orge, comparativement à ceux à base de maîs, ne modifie pas le niveau de consommation d'énergie digestible, mais réduit la vitesse de croissance $(\mathrm{P}<0,0 \mathrm{I})$. Les caractéristiques de composition corporelle ne sont pas affectées, à l'exception du rendement, plus faible arec les régimes à base d'orge $(\mathrm{P}<0,0 \mathrm{I})$.

Dans le cas particulier des animaux des lots $\mathrm{I}$ et 4 , qui ont bénéficié d'un apport satisfaisant de matières azotées, l'abaissement de la concentration en énergie du régime, obtenu par substitution de l'orge au maïs, entraîne cependant, au cours de la première phase de la croissance $(20-50 \mathrm{~kg})$, une légère augmentation du niveau de consommation d'énergie digestible $(6,24$ contre 6,13 Mcal ED/jour), chez les mâles castrés comme chez les femelles. Quoi qu'il en soit, la vitesse de croissance est sensiblement plus faible avec les régimes moyennement concentrés en énergie, de type orge-tourteau de soja, les mâles castrés réagissant plus défavorablement que les femelles ( $658 \mathrm{~g} / \mathrm{jour}$ au lieu de 720 ). En conséquence, le coût énergétique du gain (Mcal ED $/ \mathrm{kg}$ ) augmente sensiblement, surtout chez les mâles castrés $(9,77$ contre 8,74$)$, lorsque la concentration en énergie du régime est abaissée.

Les observations relatives à la période totale (20-Ioo kg de poids vif) confirment les résultats de la période $20-50 \mathrm{~kg}$ : augmentation du niveau de consommation d'énergic digestible $(+3,8$ p. Ioo), diminution de la vitesse de croissance unique- 


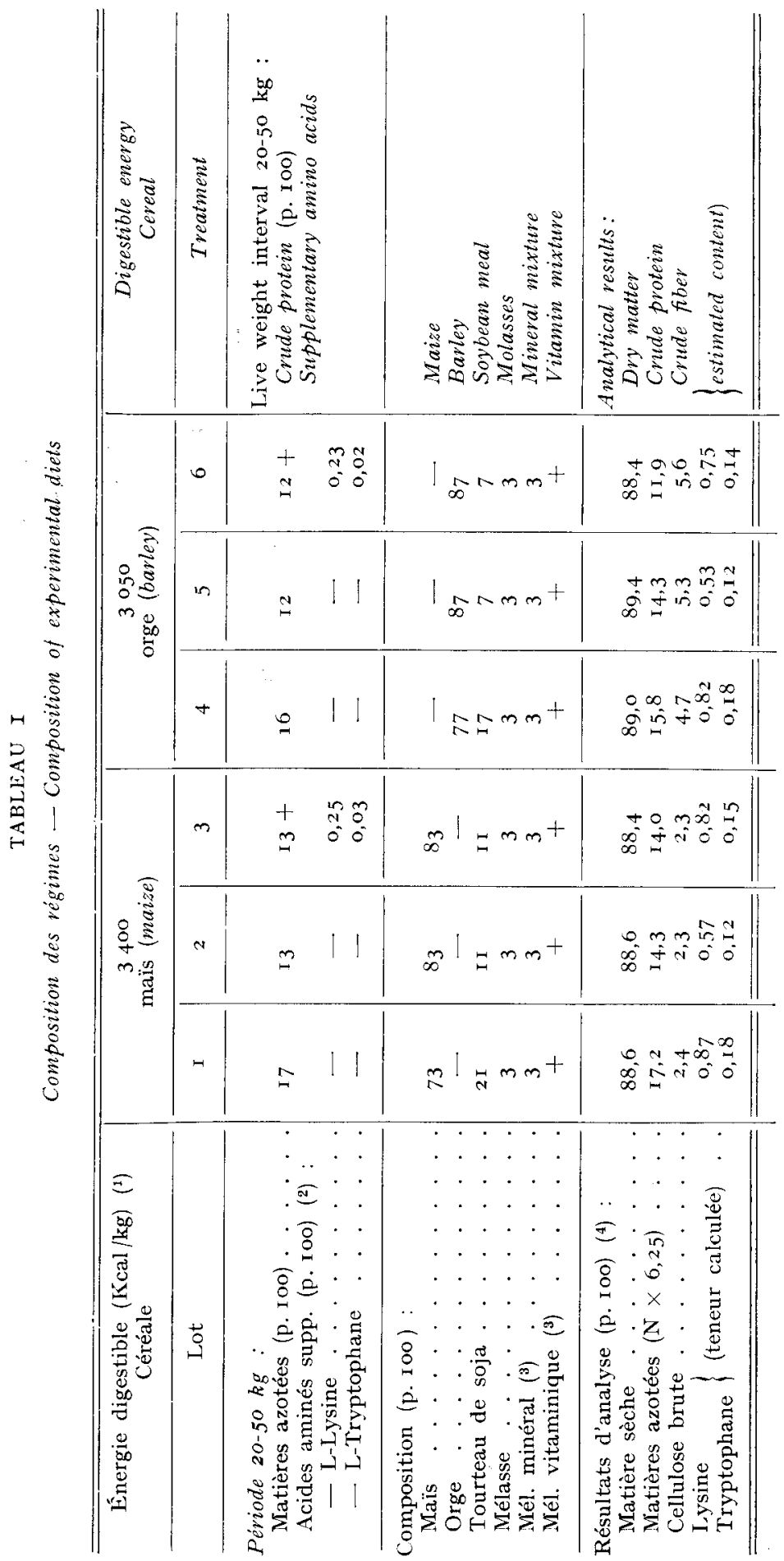




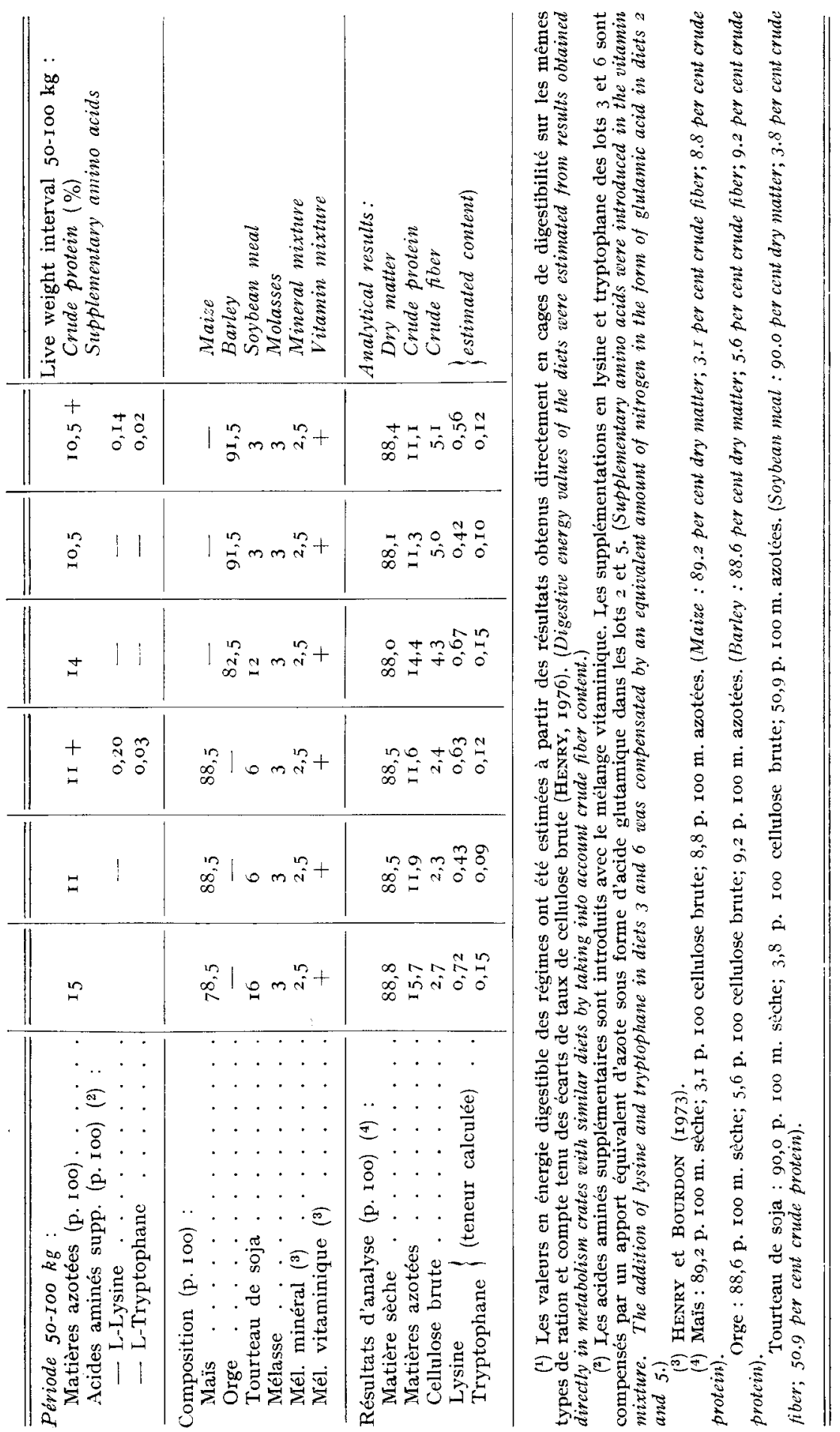




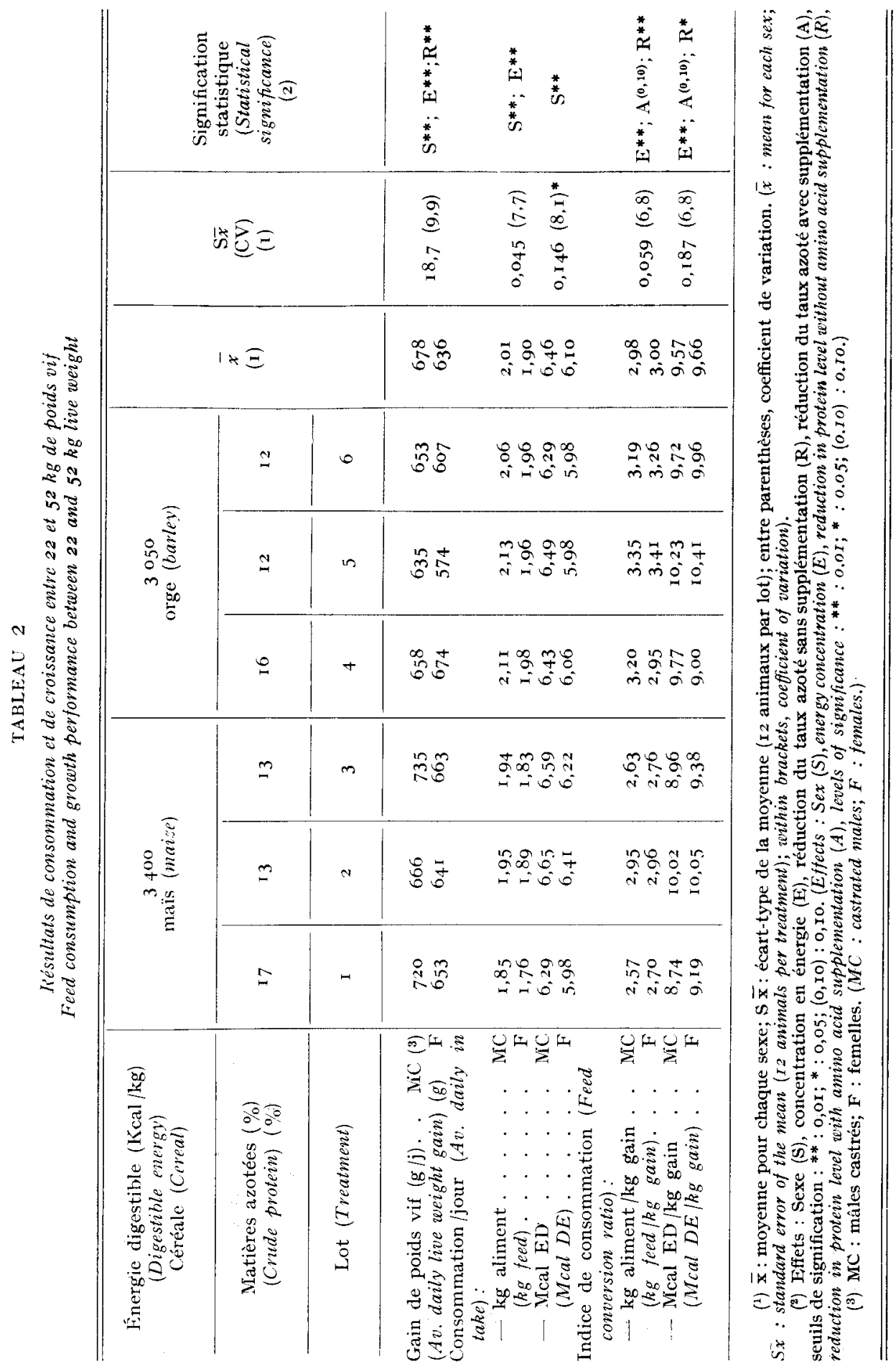



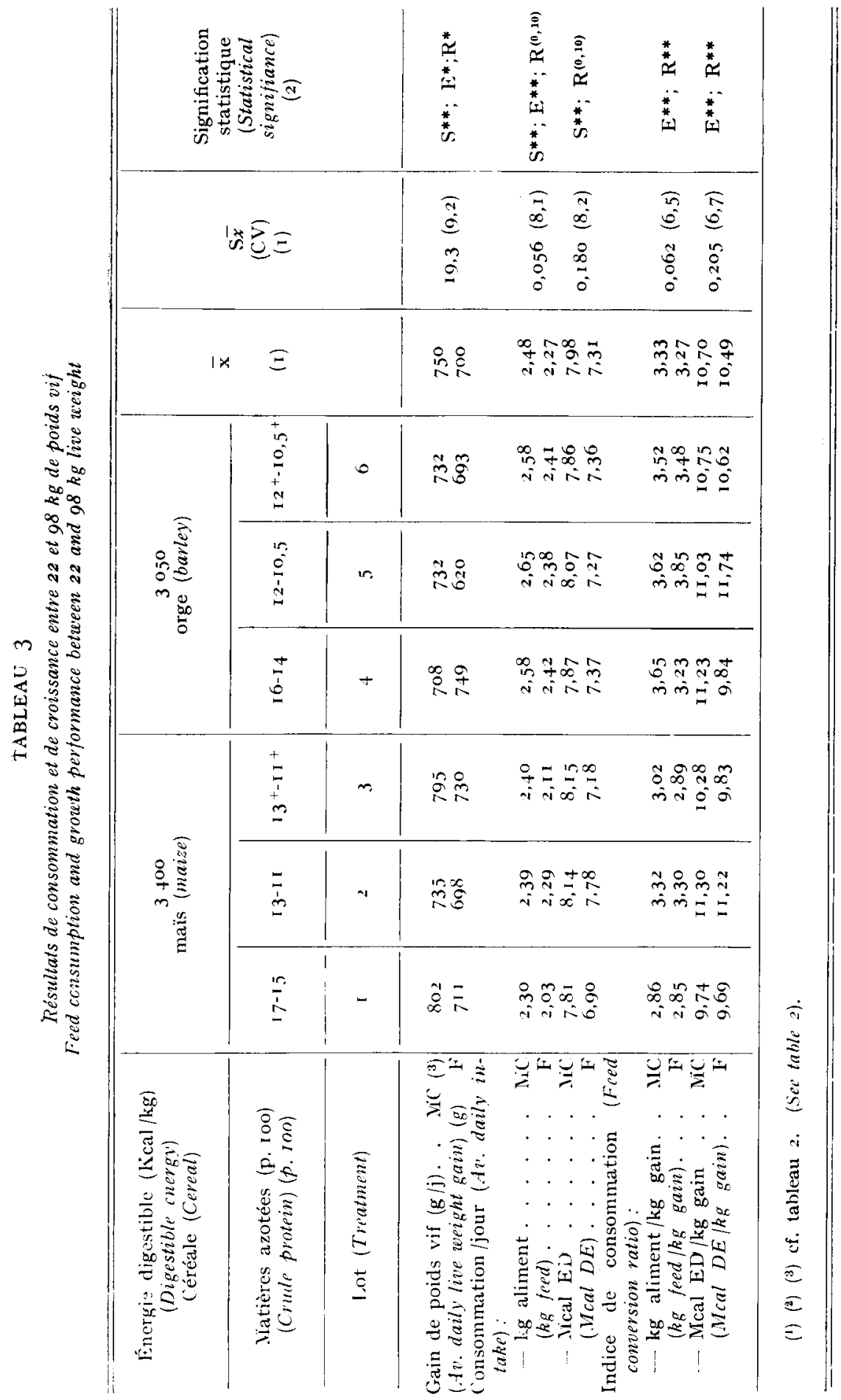
ment chez les mâles castrés (70\$ g /jour contre 802), accompagnée d'une élévation de l'indice de consommation, également chez les mâles castrés (I I,23 $\mathrm{McalED} / \mathrm{kg}$ gain contre 9.74) lors d'une réduction de Io p. Ioo de la concentration en énergie du régime.

Bien que les résultats ne soient pas significativement différents, les animaux recevant le régime à base d'orge ont tendance à être plus gras, quel que soit le sexe, le rapport longe-bardière étant de $\mathrm{I}, 82$ au lieu de 2,04 avec les régimes à base de maï:

En définitive, le remplacement du maîs par l'orge conduit à un niveau de consommation d'énergie digestible équivalent, sinon supérieur, avec cependant, pour les mâles castrés, une réduction de la vitesse de croissance, accompagnée d'une augmentation de l'indice de consommation et d'une adiposité accrue des carcasses.

\section{2. - Réduction du taux de matières azotées sans supplémentation en acides aminés}

L'examen des résultats de croissance entre 20 et $50 \mathrm{~kg}$ de poids vif fait ressortir une tendance à une différenciation de la réponse des animaux à une réduction du taux azoté selon la céréale, l'interaction n'étant toutefois pas significative. Dans le cas des régimes à base de maïs, l'abaissement du taux de matières azotées entraîne une hyperplagie compensatrice notable $(+6,4 \mathrm{p}$. Ioo), mais la vitesse de croissance est réduite et l'indice de consommation accru $(+\mathrm{I} 2,2 \mathrm{p}$. I0o: $\mathrm{P}<0,05)$. En revanche, chez les animaux recevant les régimes à base d'orge, la consommation d'aliment n'est pas modifiée par la diminution du taux de matières azotées. Il s'ensuit une diminution du gain moyen quotidien, très marquée chez les femelles, et une augmentation significative $(\mathrm{P}<0,05)$ de l'indice de consommation avec la réduction du taux de matières azotées $(+4,7$ p. Ioo pour les mâles castrés et + I5,6 p. Ioo pour les femelles).

Les mémes effets se retrouvent pendant la période totale $(20-100 \mathrm{~kg})$. I.'hyperphagie observée durant la phase initiale de la croissance dans le lot 2 , à base de mais, comparativement au lot $\mathrm{I}$, se maintient pendant la période de finition, comme le montre la figure I; l'accroissement est ainsi de 12,8 p. roo pour les femelles et de 3,9 p. Ioo pour les mâles castrés $(P<0,10)$. Les vitesses de croissance ne sont pas significativement affectées, notamment chez les femelles, mais on observe un accroissement hautement significatif de l'indice de consommation dans les deux sexes ( + I6 p. I00). Avec les régimes à base d'orge, la consommation d'énergie digestible et le gain moyen quotidien ne sont pas modifiés chez les mâles castrés. Cette observation est à relier à une croissance compensatrice marquée en finition ( 8 I I g/jour dans le lot 5 contre $746 \mathrm{~g}$ /jour dans le lot 4). Chez les femelles, au contraire, la vitesse de croissance est notablement abaissée par la réduction du taux azoté (- I7 p. IOO). Cette différence de comportement des animaux des deux sexes se répercute sur l'indice de consommation, inchangé chez les mâles castrés mais nettement augmenté chez les femelles.

Au niveau de la composition corporelle, pour l'ensemble des traitements, la. réduction du taux de matières azotées sans supplémentation en acides aminés entraîne, dans les deux sexes, une diminution du pourcentage de longe ( $\mathrm{P} \sim 0, \mathrm{OI})$, du rapport longe/bardière $(\mathrm{P}<0,05)$ et une augmentation du pourcentage de bardière $(\mathrm{P}<0,05)$, de panne $(\mathrm{P}<0,0 \mathrm{I})$, ainsi que de l'épaisseur du lard dorsal (mais non significative), sans que les critères de rendement et de longueur de carcasse soient affectés. 
RÉDUCTION DU TAUX AZOTÉ ET CROISSANCE DU PORC

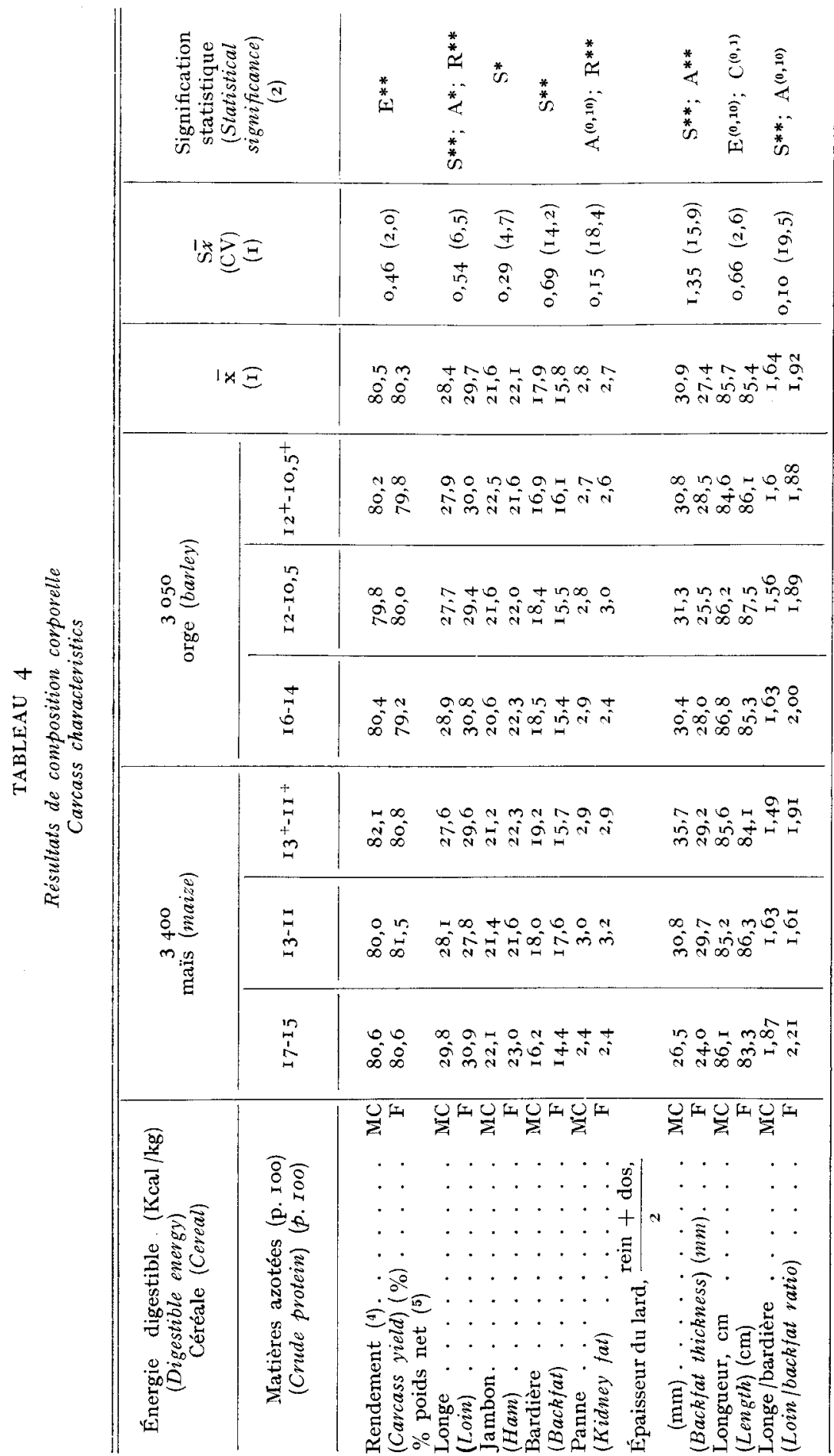

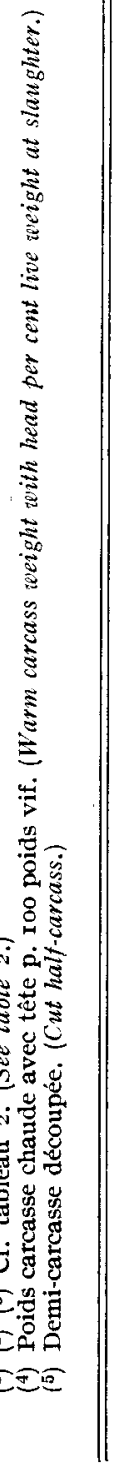


Cependant, chez les animaux recevant les régimes à base de maïs, la réduction du taux de matières azotées provoque une augmentation significative $(\mathrm{P}<0,05)$ de l'état d'engraissement quel que soit le critère considéré, en relation avec une consommation supérieure et une vitesse de croissance moindre des animaux. En revanche, avec les régimes à base d'orge, les données relatives à la composition corporelle ne sont pas significativement différentes, bien qu'allant dans le sens

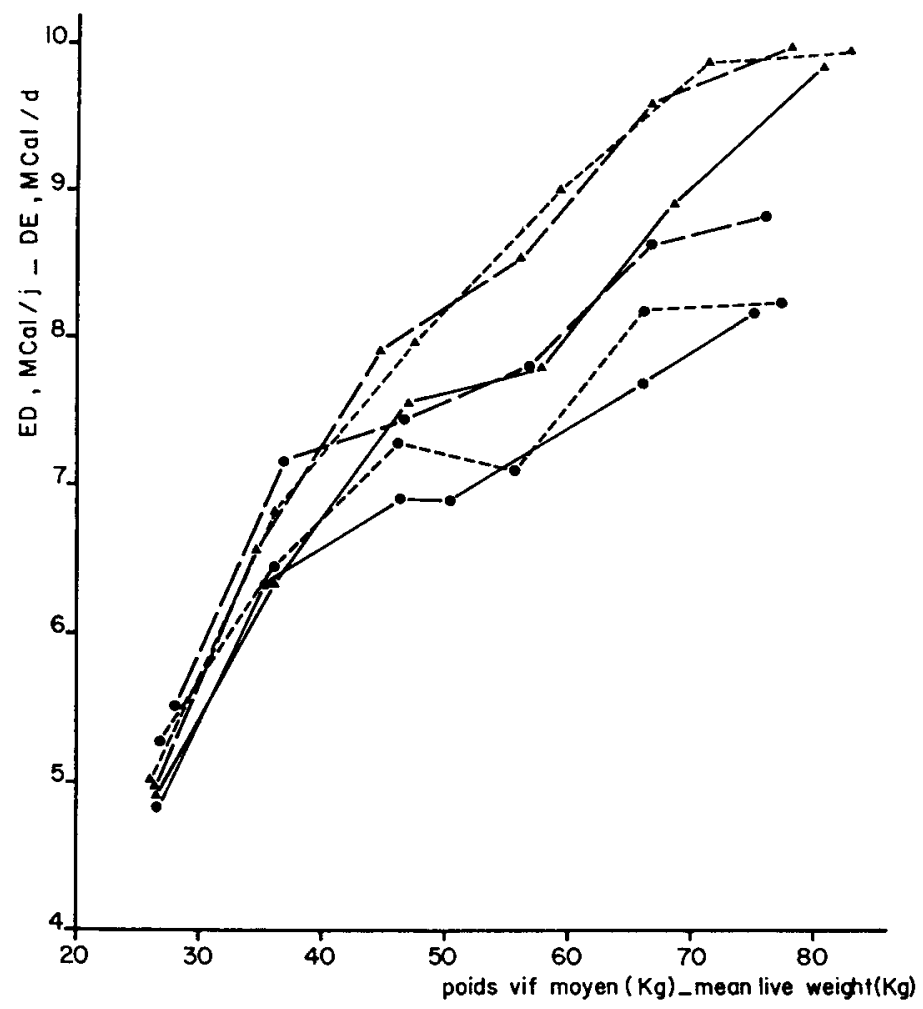

FIG. I. - Evolution de la consommation moyenne d'énergie digestible (Mcal/j) par lot en fonction du poids vif moyen tous les I 4 jours : régimes maïs-tourteau de soja.

- $\operatorname{lot} \mathrm{I} ;-\longrightarrow$ - $\operatorname{lot} 2 ;-. .-\operatorname{lot} 3 ; \Delta$ mâles castrés; • femelles

Evolution of the mean daily intake of digestible energy (Mcal) per treatment with average body weight during successive 2 week periods: maize-soybean meal diets. - treatment $\mathrm{r}$; _..... treatment 2 ; ..... treatment 3 ; $\Delta$ castrated males; $\bullet$ females

d'une adiposité accrue (diminution du pourcentage de longe) lorsque le taux azoté est abaissé.

En résumé, à la suite d'une réduction relativement importante du taux de matières azotées, les animaux réagissent différemment selon la concentration énergétique du régime dont l'effet est ici confondu avec celui de la céréale : l'hyperphagie constatée avec une ration riche en énergie (mais-tourteau de soja) ne se manifeste pas avec un régime plus encombrant (orge-tourteau de soja). Toutefois, quelle que soit la céréale utilisée, le gain moyen quotidien est abaissé, notamment 


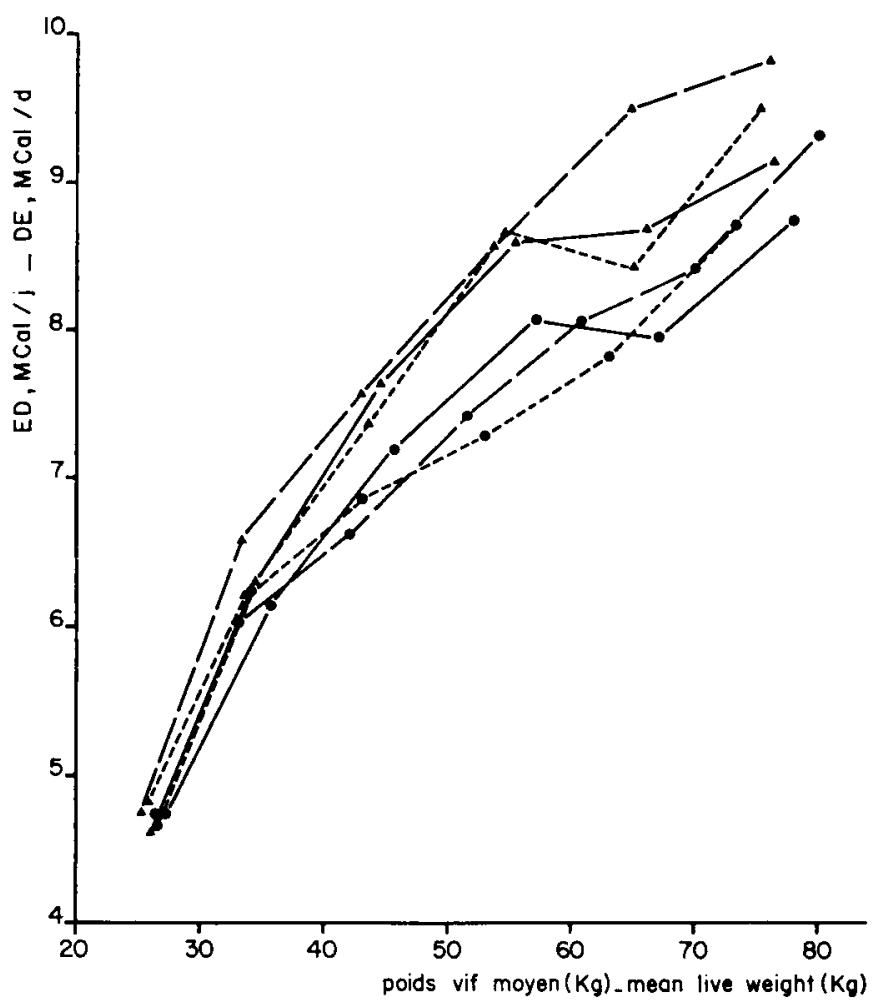

FIg. 2. - Evolution de la consommation moyenne d'énergie digestible (Mcal $/ j$ ) par lot en fonction du poids vif noyen tous les $1+$ jours: régimes orge-tourteau de soja.

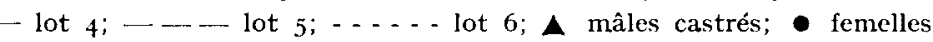

Evolution of the mean daily intake of digestible energy (Mcal)

per treatment with average body weight during successive 2 week periods: barley-soybean meal diets..

treatment $4 ; \ldots \ldots$ treatment $5 ; \ldots . .-$ treatment 6 ; $\mathbf{A}$ castrated males; $\bullet$ females.

pendant la phase initiale de la croissance, entraînant une détérioration de l'indice. de consommation et de la composition corporelle (animaux plus gras), les effets. étant plus marqués avec le régime riche en énergie.

\section{3. - Réduction du taux de matières azotées avec supplémentation en acides aminés limitants}

Entre 20 et $50 \mathrm{~kg}$ de poids vif (tabl. 2), l'hyperphagie notéc lors d'une réduction du taux de matières azotées sans supplémentation en acides aminés, se maintient après supplémentation chez les mâles castrés recevant les régimes à base. de maïs $(+4,9$ p. Ioo). Les femelles ont cependant tendance à réduire leur niveau de consommation après supplémentation (fig. I). La vitesse de croissance et l'indice. de consommation ne sont pas significativement affectés par le taux azoté lorsqueles apports d'acides aminés sont suffisants. Avec les régimes à base d'orge, les niveaux de consommation sont comparables dans les lots 4 et 6 , quel que soit le sexe. Mais les femelles accusent une diminution de la vitesse de croissance (-- II,7 p. IOO) 
et de l'efficacité alimentaire (- II, I p. IOo), sous l'effet d'une réduction du taux azoté, ce qui n'est pas le cas des mâles castrés.

Avec les régimes maïs-tourteau de soja (lots I et 3 ), les observations faites sur la période totale confirment celles effectuées entre 20 et $50 \mathrm{~kg}$. La réduction du taux azoté avec supplémentation en acides aminés provoque une hyperphagie plus marquée chez les mâles castrés que chez les femelles (fig. I), une vitesse de croissance identique ( $762 \mathrm{~g} /$ jour contre $\left.75^{6}\right)$ et un coût énergétique du gain qui tend à s'élever (10,05 McalED / $\mathrm{kg}$ de gain au lieu de 9,72). Dans le cas des régimes à base d'orge, pour des niveaux de consommation très voisins dans les lots 4 et 6 , les mâles castrés ont des vitesses de croissance et des indices de consommation comparables, les femelles ayant une croissance et une efficacité alimentaire sensiblement modifiées $(-7,5$ p. Ioo pour la vitesse de croissance et $+7,9$ p. roo pour l'indice de consommation).

Les résultats de consommation et de croissance ne semblent donc pas modifiés par une réduction du taux azoté lorsque les acides aminés sont fournis en quantité suffisante, à l'exception des femelles recevant un régime à base d'orge.

Pour l'ensemble des traitements, la réduction du taux azoté accompagnée d'une supplémentation en acides aminés entraîne, au niveau de la composition corporelle, une diminution du pourcentage de longe $(\mathrm{P}<0,05)$ et du rapport longe / bardière $(\mathrm{P}<0, \mathrm{IO})$, une augmentation de l'épaisseur moyenne du lard dorsal $(\mathrm{P}<\mathrm{O}, \mathrm{OI})$ et du pourcentage de panne $(\mathrm{P}<0, \mathrm{IO})$. Cet accroissement de l'état d'engraissement est d'ailleurs plus accentué avec les régimes à base de maïs : longe : -6, I p. I0o $(\mathrm{P}<0, \mathrm{IO})$, jambon : - 3, I p. Ioo (effet non significatif), bardière : + I3,7 p. Ioo $(\mathrm{P}<0, \mathrm{IO})$, panne : $+20,8$ p. Ioo $(\mathrm{P}<0, \mathrm{IO})$, épaisseur moyenne du lard dorsal : 28,4 p. Ioo $(\mathrm{P}<0,05)$, rapport longe /bardière : - I6,6 p. Ioo $(\mathrm{P}<\mathrm{o}, \mathrm{IO})$. Par contre, avec les régimes orge-tourteau de soja, l'adiposité des carcasses n'est pas modifiéc par la réduction du taux azoté, les femelles étant toutefois légèrement plus grasses. Ces observations rejoignent d'ailleurs celles déjà faites pour les performances de croissance.

En définitive, la réduction du taux de matières azotées dans les rations à base de maïs, accompagnée d'une supplémentation en acides aminés limitants (lysine et tryptophane dans la présente étude), n'a pas entraîné de retard de croissance ni d'augmentation de l'indice de consommation, mais la surconsommation des animaux recevant ce type de régime riche en énergie aboutit à la production de carcasses plus grasses. Par contre, avec les régimes orge-tourteau de soja, la réduction des normes azotées quantitatives semble sans conséquence défavorable sur la qualité des carcasses, lorsque l'apport d'acides aminés indispensables est maintenu à son niveau optimum.

\section{Discussion}

\section{I. - Effets comparés de l'orge et du maïs}

Les données de la bibliographie, relatives à la comparaison de régimes à base d'orge et de maïs, en alimentation à volonté et généralement sous forme de farine, lorsque le rapport matières azotées /énergie est maintenu constant, font apparaître des performances moindres avec l'orge sur le plan tant de la vitesse de croissance que du niveau d'ingestion énergétique, le coût énergétique du gain étant pratiquement inchangé. Il en résulte une adiposité réduite des carcasses (GREER et al., 
I965; Gill, OldField et EnGland, i966). Il en est d'ailleurs de même sous l'effet d'une élévation du taux de cellulose dans la ration (HENRY, I969). La divergence apparente entre nos résultats et ceux rapportés dans la bibliographie peut être expliquée par la différence de mode de présentation de l'aliment (farine ou granulé). Ainsi, selon REIMER et MEADE (I964), les performances obtenues avec l'orge et le maïs sont sensiblement équivalentes lorsque les aliments sont distribués sous forme de granulés, mais elles sont significativement inférieures avec les régimes à base d'orge, lorsque l'aliment est présenté en farine. Toutefois, dans des conditions expérimentales similaires à celles décrites et en l'absence de différences dans les consommations d'énergie digestible entre l'orge et le maïs, SEllier et al. (I974) signalent une adiposité supérieure dans le cas de régimes à base de maïs. On pourrait invoquer également une imprécision dans l'estimation de la valeur énergétique des céréales, notamment de l'orge, quoi qu'elle ait été obtenue à partir de régimes similaires (HENRY, I976). En tout état de cause, l'élévation du coût énergétique du gain (en énergie digestible), avec l'orge, peut résulter d'un rendement plus faible de l'utilisation de l'énergie métabolisable par rapport à celle du maïs (THoRBEK, r975) et/ou de l'accroissement de l'état d'engraissement des carcasses, lui-même en relation avec une consommation d'énergie supérieure.

\section{2. - Influence de la réduction globale du taux de matières azotées}

Les résultats obtenus confirment les observations concernant les effets d'une réduction du taux azoté, sans supplémentation en acides aminés, à partir d'un niveau " normal ": diminution de la vitesse de croissance, augmentation de l'indice de consommation et de l'état d'engraissement des carcasses (JuRGENs et al., I967; Henry et Rerat, i972; Stahly et Wahlstrom, I973; Wahlstrom et Libal, r974). Cependant, les données restent relativement contradictoires pour ce qui est des effets sur le niveau de consommation. Certains auteurs (Clawson, I967; BAKER, KATZ et EASTER, I975) constatent qu'une réduction modérée du taux azoté s'accompagne d'une élévation de la quantité d'aliment consommée, ce qui est le cas des régimes maïs-tourteau de soja dans notre étude. Lors de la distribution d'un régime hypoazoté, il a été d'ailleurs montré que le porc, comme le rat, a tendance à manifester une hyperphagie compensatrice pour essayer de satisfaire son besoin azoté (RERAT et HENRY, I964; Henry et RERAT, I965). D'autres travaux (Stahly et Wahlstrom, I973; Sharda, Mahan et Wilson, I976), portant exclusivement sur des régimes maïs-tourteau de soja, montrent que la consommation d'aliment est équivalente, sinon inférieure, lorsque le taux azoté est abaissé, ce qui est le cas des régimes orge-tourteau de soja dans cette expérience. Ces contradictions ont, en partie, pour origine la grande diversité des taux azotés $€$ t des teneurs en acides aminés des régimes utilisés. La réponse différente des animaux selon le sexe, qui se manifeste par une plus grande sensibilité des femelles à la réduction du taux azoté et des apports d'acides aminés, comparativement aux mâles castrés, a été retrouvée antérieurement dans notre laboratoire (HENRY et RERAT, I972).

\section{3. - Influence de la réduction du taux d'azote indifférencié}

L'objectif principal de cette expérience était de préciser l'influence d'une réduction du taux de matières azotées sur les performances de croissance et la composition corporelle des animaux, les besoins en acides aminés indispensables étant 
couverts par ailleurs. Les données de la bibliographie, recueillies exclusivement sur des régimes de type maïs-tourteau de soja indiquent, pour l'aliment à taux azoté réduit mais équilibré en acides aminés, soit une surconsommation des animaux (KATz et al., I973; BAKER, KATz et EASTER, I975), soit un niveau de consommation équivalent (SHARDA, MAHAN et Wilson, I976), les performances de croissance et l'efficacité alimentaire étant généralement détériorées, parallèlement à une accentuation de l'état d'engraissement des animaux (WAHLSTRom et LibaL, I974). Ces observations sont confirmées par les résultats de la présente étude, notamment avec le maïs. Ceci montre que le taux d'azote indifférencié dans la ration n'a pas permis d'optimiser les performances de croissance dans les conditions d'équilibre en acides aminés indispensables et a, de ce fait, entraîné une augmentation du niveau de consommation. A l'inverse, l'élévation du taux global de matières azotées, avec des régimes apportant les mêmes quantités d'acides aminés indispensables et pour des rétentions azotées équivalentes, provoque une réduction de l'état d'engraissement des carcasses (Sharda, Mahan et Wilson, r976), ce qui corrobore les faits généralement rapportés dans les conditions du rationnement (CoOke, Longe et Lewis, I972; Wenk, Pfirter et Schurch, I976).

Il est à noter que la surconsommation constatée avec les régimes à taux azoté réduit, masque les effets propres du taux azoté, en raison d'un apport énergétique excessif qui n'apparaît pas dans les conditions du rationnement (HExRY, I977). La réponse différentielle des animaux (au niveau de la consommation) lors d'une réduction du taux azoté, selon la céréale utilisée, semble, pour le moment, difficile à interpréter. D'une part, la dilution de l'énergie dans le cas de l'orge peut constituer un facteur limitant de la capacité d'ingestion des animaux. D'autre part, dans ce type de régime, un autre acide aminé limitant, probablement la thréonine, pourrait induire une limitation du niveau de consommation. Cette dernière hypothèse semble confirmée par le comportement des femelles du lot 6 , pénalisées sévèrement au niveau de la croissance et de l'indice de consommation, étant entendu qu'elles sont plus sensibles aux apports azotés qualitatifs et quantitatifs que les mâles castrés (HENRY et RERAT, I972). De plus, elles semblent mieux en mesure d'ajuster leur niveau de consommation en fonction de la qualité des apports alimentaires. C'est ainsi que l'hyperphagie consécutive à un abaissement du taux de matières azotées est beaucoup moins marquée après rééquilibre en acides aminés de la ration, contrairement à ce que l'on observe chez les mâles castrés.

En d'autres termes, les variations du niveau d'ingestion de nourriture, selon l'équilibre azote-énergie du régime, produisent des effets différents chez les mâles castrés et les femelles. Alors que chez les mâles castrés, l'élévation du niveau d'ingestion a surtout pour effet de fournir davantage d'énergie pour la formation des dépôts gras, chez les femelles, par contre, ce sont les apports supplémentaires d'acides aminés qu'elle procure qui permettent un accroissement des masses maigres. Il est vraisemblable que les mêmes différences s'observent en fonction des types génétiques, si l'on se réfère par exemple aux résultats enregistrés sur des porcs présentant des aptitudes variables sur le plan du développement musculaire (SEllier et al., I974; Bolet et al., I977).

En conclusion, une réduction des normes azotées quantitatives compensée par un apport supplémentaire d'acides aminés limitants, en alimentation à volonté. implique que le niveau de consommation soit parfaitement maîtrisé, pour éviter notamment un phénomène d'hyperphagie compensatrice, responsable d'une augmentation de l'état d'engraissement des carcasses à l'abattage, principalement chez les animaux recevant des régimes à forte concentration en énergic, du type maïs-tourteau de soja. Il semble cependant, que cette maîtrise du niveau de consom- 
mation soit plus facilement réalisable dans le cas des régimes moyennement concentrés en énergie, de type orge-tourteau de soja. Une étude complémentaire est en cours pour dissocier l'effet de la dilution de l'énergie de la ration de celui de la céréale, compte tenu de sa composition particulière en acides aminés.

Accepté pour publication en juin r977.

\title{
Summary
}

\author{
Effects of a reduction in the dietary protein level \\ in the growing pig, on feed intake and growth performance, \\ according to amino acid balance and cnergy concentration
}

An experiment was performed on 72 growing Large White pigs between 20 and roo $\mathrm{kg}$ live weight, in order to study the influence of reducing the level of dietary protein on feed intake, growth performance and carcass characteristics, according to amino acid balance and energy concentration. Six groups of 12 animals in each (half castrated males and half females) were compared according to $2 \times 3$ factorial design:

- 2 levels of dietary energy: 3400 and 3050 Kcal digestible energy (DE) $/ \mathrm{kg}$ air-dry feed, with diets based on maize and barley, respectively, in combination with soybean meal, so that dietary energy concentration was confounded with cereal source,

- 3 levels of protein: a normal level, corresponding to the usual recommendations, on the basis of digestible crude protein per Mcal DE $(43.5 \mathrm{~g}$ up to $50 \mathrm{~kg}$ live weight and $39.0 \mathrm{~g}$ afterwards) and two reduced levels $(-25 \%)$, with or without supplementary lysine and tryptophan to meet the requirements for primary and secondary limiting amino acids (Table I).

In all treatments, the pigs were individually fed ad libitum pelleted diets $(5 \mathrm{~mm}$ diameter). They were weighed every two weeks and after slaughter, at Ioo kg live weight, backfat measurements were taken and carcasses were evaluated for lean and fat cuts.

As shown in Tables 2 and 3 , the daily $D E$ intake was not significantly affected after replacing maize by barley in the diet (7.62 Mcal DE/day vs 7.26 during the whole period of growth), but in castrated males average daily gain (ADG) was reduced $(-12,5 \%)$ and feed conversion ratio (FCR) was increased (+ I \% \%). In both sexes, carcass adiposity was higher with barley diets (loin/back fat ratio: I.8 os 2.04; ham + loin: 5 I .3 vs $52.9 \%$ ).

The overall reduction of protein level caused a compensatory hyperphagia (fig. I and 2) in pigs fed maize diets $(+8.5 \% \mathrm{DE}$ intake per day). With both cereals, however, a reduction in ADG was observed $(-5.5$ and $-7.0 \%$ with maize and barley, respectively). This resulted in a deterioration of FCR, which was mostly apparent with maize diets (- I $6 \%)$, whereas carcass fatness was significantly increased (loin/back fat ratio: 1.62 vs 2.04 and I.72 vs I.85, with maize and barley diets, respectively).

The overconsumption of feed which was observed in pigs fed maize diets with a low level of protein was maintained after supplementing with limiting amino acids (fig. I), but the effect was less pronounced in females $(+2 \%)$ than in castrated males $(+5.5 \%)$. As compared to the pigs fed a normal protein level in the diet, ADG was not altered by a reduced protein level, for a corrected amino acid balance. But FCR was decreased $(-3.5 \%)$ and carcass adiposity was significantly increased (loin / back fat ratio: r.70 vs 2.04). With barley diets, on the contrary, a reduction in the content of non essential nitrogen did not seem to affect daily feed intake (7.6r $v /$ 7.62 Mcal DE per day), ADG (7I2 vs $728 \mathrm{~g}$ ) and carcass characteristics. A differential response was observed according to sex. Females were more sensitive than castrated males to variation in quantitative and qualitative protein supply for lean deposition. Castrated males, on the contrary, were severely affected by a decrease in dietary energy concentration, with regard to growth performance and feed efficiency, since less energy was used for fat deposition.

\section{Références bibliographiques}

BAKER D. H., KAtz R. S., EAster R. A., I975. Lysine requirement of growing pigs at two levels of dietary protein. J. Anim. Sci., 40, 85 T-856.

Bolet G., Desmoulin B., Sellier P., Henry Y., 1977. Interactions entre type génétique, sexe et conditions nutritionnelles chez le porc en croissance. Journées Rech. Porcine en France, I.N.R.A.-1.T.P. éd. Paris, 79-84. 
Clawson A. J., I967. Influence of protein level, amino acid ratio and caloric density of the diet on feed intake and performance of pigs. J. Anim. Sci., 26, 328-334.

Cooke R., LoDGe G. A., Lewis D., I972. Influence of energy and protein concentration in the diet on the performance of growing pigs. 3. Response to differences in levels of both energy and protein. Anim. Prod., 14, $219-223$.

Gill D. R., Oldfield J. E., England D. C., i 966 . Comparative values of hulless barley, regular barley, corn and wheat for growing pigs. J. Anim. Sci., 25, 34-36.

Greer S. A. N., Hays V. W., Speer V. C., Mc Call J. T., Hammond E. G., ig65. Effects of level of corn and barley-base diets on performance and body composition of swine. J.Anim. Sci., 24, IOO8-IOI3.

HENRY Y., RERAT A., I965. Ingestion spontanée d'éléments énergétiques en régimes mixtes et séparés chez le rat en croissance. Ann. Biol. anim. Bioch. Biophys., 5, 283-292.

Henry Y., 1969. Effets nutritionnels de l'incorporation de cellulose purifiée dans le régime du porc en croissance-finition. II. Influence sur les performances de croissance et la composition corporelle. Ann. Zootech., 18, 371-384.

Henry Y., Rerat A., I972. La relation azote-énergie dans l'alimentation du porc en croissance. IXth Internat. Congress of Nutrition, Mexico.

Henry Y., Bourdon D., I973. Utilisation digestive de l'énergie et des matières azotées de la fèverole sous forme entière ou décortiquée, en comparaison avec le tourteau de soja. Journées Rech. Porcine en France, I.N.R.A.-I.T.P. éd. Paris, Io5-I I 4.

Henry Y., I976. Prediction of energy values of feeds for swine from fiber content. Ist Internat. Symp. Feed Composition, Animal Nutrition Requirements, and Computerization of Diets, Utah State Univ., Logan, Utah (U.S.A.).

Henry Y., Pion R., Rerat A., I976. Protein supplies for pigs and possibilities of reducing protein feeding standards. World Rev. Anim. Prod., 12, 9-32.

HENRY Y., I977. Influence du taux azoté sur les performances du porc en finition. Journées Rech. Porcine en France, I.N.R.A.-I.T.P. éd. Paris, 259-264.

Jurgens M. H., Hudman D. B., Adams C. H., Peo E. R., I967. Influence of a dietary supplement of lysine fed at two levels of protein on growth, feed efficiency and carcass characteristics of swine. J. Anim. Sci., 26, 323-327.

Katz R. S., Baker D. H., Sasse C. E., Jensen A. H., Harmon B. G., i973. Efficacy of supplemental lysine, methionine and rolled oats, for weanling pigs fed a low protein corn soybean meal diet. J. Anim. Sci., 37, I165-I 168.

Reimer D., Meade R. J., I964. Barley rations for swine. I. Influence of source and level of dietary protein on rate and efficiency of gain of growing swine. J. Anim. Sci., 23, 392396.

RERAT A., Henry Y., I964. Consommation spontanée d'énergie en alimentation mixte et séparée chez le porc en croissance. Ann. Biol. anim. Bioch. Biophys., 4, 44I-444.

Sellier P., Houix Y., Desmoulin B., Henry Y., i974. Premières observations sur la relation entre conditions nutritionnelles et type génétique chez des porcs femelles. Journées Rech. Porcine en France, I.N.R.A.-I.T.P. éd. Paris, 209-2 I9.

Sharda D. P., Mahan D. C., Wilson R. F., 1976. Limiting amino acids in low protein cornsoybean meal diets for growing-finishing swine. J.Anim. Sci., 42, II 75-I I 8 I.

Stahly T. S., Wahlstrom R. C., I973. Effects of dietary protein level and feed restriction on performance and carcass characteristics of swine. J. Anim. Sci., 36, I Io9-III3.

Thorbek G., 1975. Studies on energy metabolism in Growing Pigs, Beretning Statens Husbyrbrugs Fors $\varnothing g, 424,157$.

Wahlstrom R. C., Libal G. W., I974. Gain, feed efficiency and carcass characteristics of swine fed supplemental lysine and methionine in corn-soybean meal during the growing and finishing periods. J. Anim. Sci., 38, I26r-1 265.

Wenk C., Pfirter H. P., Schürch A., I976. Zur Energie und Proteinversorgung das Mastschweines. Z. Tierphysiol. Tierernähr. Futtermittelk., 36, 249-266. 